\title{
Teleparallel equivalent of non-Abelian Kaluza-Klein theory
}

\author{
A. L. Barbosa, ${ }^{*}$ L. C. T. Guillen, ${ }^{\dagger}$ and J. G. Pereira ${ }^{\ddagger}$ \\ Instituto de Física Teórica, Universidade Estadual Paulista, Rua Pamplona 145, 01405-900 São Paulo SP, Brazil
}

(Received 22 July 2002; published 30 September 2002)

\begin{abstract}
Based on the equivalence between a gauge theory for the translation group and general relativity, a teleparallel version of the non-Abelian Kaluza-Klein theory is constructed. In this theory, only the fiber-space turns out to be higher dimensional, spacetime being kept always four dimensional. The resulting model is a gauge theory that unifies, in the Kaluza-Klein sense, gravitational and gauge fields. In contrast with the ordinary Kaluza-Klein models, this theory defines a natural length scale for the compact submanifold of the fiber space, which is shown to be of the order of the Planck length.
\end{abstract}

DOI: 10.1103/PhysRevD.66.064028

PACS number(s): 04.50.+h, 12.10.-g

\section{INTRODUCTION}

In ordinary Kaluza-Klein theories [1], the geometrical approach of general relativity is adopted as the paradigm for the description of all other interactions of nature. In the original Kaluza-Klein theory, for example, gravitational and electromagnetic fields are described by a Hilbert-Einstein Lagrangian in a five-dimensional spacetime. In the case of the non-Abelian gauge theory, the unification with gravitation, a possibility first raised in the 1960s [2], was achieved by extending the usual four-dimensional spacetime to a $(4+D)$-dimensional spacetime [3-6], with $D$ the dimension of the compact part of spacetime. According to this construction, the isometries of the $D$-dimensional compact submanifold yield the non-Abelian gauge transformations.

On the other hand, it is widely known that, at least macroscopically, general relativity is equivalent to a gauge theory [7] for the translation group [8], provided a specific choice of parameters be made [9]. In this theory, known as the teleparallel equivalent of general relativity, the fundamental field is the Weitzenböck connection, a connection presenting torsion, but no curvature. Differently from general relativity, in which gravitation is attributed to curvature, teleparallel gravity attributes gravitation to torsion. Furthermore, whereas in general relativity curvature is used to geometrize the gravitational interaction, in teleparallel gravity torsion plays the role of a gravitational force [10]. This agrees with the fact that, in any gauge theory, the classical interaction is always described by a force equation.

Now, the equivalence alluded to above opens new perspectives for the study of unified theories. In fact, instead of using the geometrical description of general relativity, we can adopt the gauge description as the basic paradigm, and in this way construct what we call the teleparallel equivalent of Kaluza-Klein models. According to this approach, both gravitational and non-Abelian gauge fields turn out to be described by a gauge-type Lagrangian. This means that, instead of obtaining the Yang-Mills construction from geometry, as is usually done in ordinary Kaluza-Klein models, in

\footnotetext{
*Electronic address: analucia@ift.unesp.br

†Electronic address: 1ctorres@ift.unesp.br

†Electronic address: jpereira@ift.unesp.br
}

the teleparallel Kaluza-Klein the geometry (that is, gravitation) is obtained from a generalized gauge model. By following this approach, a teleparallel equivalent of the Abelian Kaluza-Klein theory has already been constructed [11].

By adopting the gauge description as the basic paradigm, the purpose of this paper will be to use the equivalence between general relativity and teleparallel gravity to construct the teleparallel equivalent of a non-Abelian Kaluza-Klein theory. In other words, instead of extending spacetime to higher dimensions, it is the internal (fiber) space that will be extended to $(4+D)$ dimensions, spacetime being kept always four dimensional. Similarly to the ordinary nonAbelian Kaluza-Klein model, the gauge transformations will be obtained from the isometries of the fiber space. This construction will be achieved through the following steps. In Sec. II, from the analysis of the dynamics of a particle submitted to both gravitational and Yang-Mills type fields, the unified gauge potentials and field strengths are defined. In Sec. III the corresponding gauge transformations are obtained from the isometries of the fiber space, and in Sec. IV, the unified gauge Lagrangian is constructed. The coupling of matter fields with the unified gauge potential is studied in Sec. V, where the explicit dependence of all dynamical variables on the internal coordinates is examined. Finally, in Sec. VI, the basic properties of the model are discussed. In particular, it is pointed out that the teleparallel Kaluza-Klein model defines a natural length scale for the compact $D$-dimensional sub-manifold of the fiber space, which is found to be of the order of the Planck length.

\section{PARTICLE DYNAMICS AND UNIFIED GAUGE POTENTIALS}

In what follows, the greek alphabet $\mu, \nu, \rho, \ldots$ $=0, \ldots, 3$ will be used to denote indices related to spacetime. According to the gauge construction, at each point of spacetime there is a fiber space, which in our case will be a $(4+D)$-dimensional space given by the direct product $M^{4}$ $\otimes B^{D}$, where $M^{4}$ is the tangent Minkowski space, and $B^{D}$ is the group manifold associated to a Yang-Mills symmetry. The first part of the latin alphabet $a, b, c, \ldots=0, \ldots, 3$ will be used to denote indices related to the Minkowski (or external) part of the fiber, whereas the second part of the latin alphabet $m, n, \ldots=5, \ldots, 4+D$ will be used to denote in- 
dices related to the Yang-Mills (or internal) part of the fiber. The second part of the latin capital alphabet will be used to denote the whole set of indices of the internal space, which runs through the values $M, N, \ldots=0, \ldots, 3,5, \ldots 4+D$. The metric of the $(4+D)$-dimensional internal space $M^{4}$ $\otimes B^{D}$ is

$$
\gamma_{M N}=\left(\begin{array}{cc}
\eta_{a b} & 0 \\
0 & \gamma_{m n}
\end{array}\right)
$$

where $\eta_{a b}$ is the metric of $M^{4}$, which is chosen to be $\eta_{a b}$ $=\operatorname{diag}(+1,-1,-1,-1)$, and $\gamma_{m n}$ is the metric of the $D$-dimensional space $B^{D}$. In general, $B^{D}$ is a (compact) curved Riemannian space, with $\gamma_{m n}=\gamma_{m n}\left(x^{m}\right)$ a function of the coordinates $x^{m}$ of $B^{D}$.

As already said, gravitation will enter as a gauge theory for the translation group, whose action will take place in the Minkowski space $M^{4}$. As the dimension of the translation group is the same as that of the Minkowski space, the first part of the latin alphabet $a, b, c, \ldots=0, \ldots, 3$ will also be used to denote the indices related to the translation group. The intermediary latin alphabet $i, j, k=5, \ldots, 4+I$ will be used to denote indices related to the Yang-Mills gauge group, with $I$ denoting the number of generators of the associated Lie algebra. The latin capital letters $A, B, C, \ldots$ $=0, \ldots, 3,5, \ldots, 4+I$ will be used to denote the whole set of indices related to the group generators. Notice that the dimension $4+D$ of the fiber does not need to coincide with the dimension $4+I$ of the gauge group.

We denote by $A^{a}{ }_{\mu}$ the gauge potential related to translations, and by $A_{\mu}^{i}$ the Yang-Mills type gauge potentials. According to the gauge description of interactions, the action integral describing a particle of mass $m$ and gauge charge $q_{i}$, under the influence of both a gravitational and a gauge field is

$$
S=\int_{a}^{b}\left[-m c d \sigma-\frac{1}{c}\left(m A^{a}{ }_{\mu} u_{a}+g A_{\mu}^{i} q_{i}\right) d x^{\mu}\right],
$$

where $d \sigma=\left(\eta_{a b} d x^{a} d x^{b}\right)^{1 / 2}$ is the Minkowski invariant interval, $u_{a}=d x_{a} / d \sigma$ is the tangent space four-velocity, and $q_{i}$ is the Noether charge related to the internal gauge transformation [12]. Notice that the mass $m$ appears as the gravitational coupling constant, whereas the gauge coupling constant is denoted by $g$. Notice, furthermore, that we are assuming the weak equivalence principle, and equating the inertial and gravitational masses.

The equation of motion following from the action (2) is

$$
\begin{aligned}
e^{a}{ }_{\mu} \frac{d u_{a}}{d s}= & \frac{1}{c^{2}} F_{\mu \nu}^{a} u_{a} u^{\nu}+\frac{g}{m c^{2}}\left(\partial_{\mu} A_{\nu}^{i}-\partial_{\nu} A_{\mu}^{i}\right) q_{i} u^{\nu} \\
& -\frac{g}{m c^{2}} A^{i}{ }_{\mu} \frac{d q_{i}}{d s}
\end{aligned}
$$

where

$$
F_{\mu \nu}^{a}=\partial_{\mu} A_{\nu}^{a}-\partial_{\nu} A_{\mu}^{a}
$$

is the gravitational field strength, $d s=\left(g_{\mu \nu} d x^{\mu} d x^{\nu}\right)^{1 / 2}$ is the spacetime invariant interval, $u^{\nu}=d x^{\nu} / d s$ is the spacetime four-velocity, and $e^{a}{ }_{\mu}$ is the tetrad field

$$
e_{\mu}^{a}=\partial_{\mu} x^{a}+c^{-2} A_{\mu}^{a} .
$$

We remark that, whereas the Minkowski indices $a, b, c, \ldots$ are raised and lowered with the Minkowski metric $\eta_{a b}$, the spacetime indices $\mu, \nu, \rho, \ldots$ are raised and lowered with the Riemannian metric

$$
g_{\mu \nu}=\eta_{a b} e^{a}{ }_{\mu} e_{\nu}^{b}
$$

which is used to write the spacetime invariant interval $d s$.

Considering now that the gauge charge $q_{i}$ satisfies the Wong equation [13]

$$
\frac{d q_{i}}{d s}+\frac{g}{\hbar c} f_{i j k} A_{\mu}^{j} q^{k} u^{\mu}=0
$$

with $f_{i j k}$ the structure constants of the gauge group, the equation of motion (3) can be rewritten in the form

$$
e_{\mu}^{a} \frac{d u_{a}}{d s}=\frac{1}{c^{2}} F_{\mu \nu}^{a} u_{a} u^{\nu}+\frac{g}{m c^{2}} F_{\mu \nu}^{i} q_{i} u^{\nu},
$$

where

$$
F_{\mu \nu}^{i}=\partial_{\mu} A^{i}{ }_{\nu}-\partial_{\nu} A^{i}{ }_{\mu}+\frac{g}{\hbar c} f^{i}{ }_{j k} A^{j}{ }_{\mu} A^{k}{ }_{\nu}
$$

is the gauge field strength. In the absence of Yang-Mills field, the equation of motion (8) can be shown to reduce to the geodesic equation of general relativity [10].

The gauge structure of teleparallel gravity allows the definition of a unified gauge potential $\mathcal{A}^{A}{ }_{\mu}$, which is assumed to have the same dimension of the gravitational potential $A^{a}{ }_{\mu}$. Consequently, the internal gauge potential $A^{i}{ }_{\mu}$ must appear multiplied by an appropriate dimensional factor, which we write in the form

$$
\mathcal{A}_{\mu}^{A} \equiv\left(A_{\mu}^{a}, \widetilde{A}_{\mu}^{i}\right)=\left(A_{\mu}^{a}, \frac{g}{\kappa} A_{\mu}^{i}\right),
$$

where $\kappa$ is a parameter to be determined later. Consequently, if we define a generalized $(4+I)$ Noether charge $p_{A}$ $=m c u_{A}$, with

$$
u_{A} \equiv\left(u_{a}, \tilde{u}_{i}\right)=\left(u_{a}, \frac{\kappa}{m} q_{i}\right)
$$

a generalized $(4+I)$ "velocity," the action (2) can be rewritten in the form

$$
S=\int_{a}^{b}\left[-m c d \sigma-\frac{1}{c^{2}} \mathcal{A}_{\mu}^{A} p_{A} d x^{\mu}\right]
$$

In the same way, we can define now a generalized field strength, 


$$
\mathcal{F}_{\mu \nu}^{A} \equiv\left(F_{\mu \nu}^{a}, \widetilde{F}_{\mu \nu}^{i}\right)=\left(F_{\mu \nu}^{a}, \frac{g}{\kappa} F_{\mu \nu}^{i}\right) .
$$

With these definitions, the equation of motion (8) assumes the form

$$
e^{a}{ }_{\mu} \frac{d u_{a}}{d s}=c^{-2} \mathcal{F}_{\mu \nu}^{A} u_{A} u^{\nu}
$$

This is the unified - gravitational plus Yang-Mills — analog of the electromagnetic Lorentz force. Its solution determines the trajectory of the particle under the influence of both gravitational and Yang-Mills fields.

\section{GENERALIZED GAUGE TRANSFORMATIONS}

A point in the $(4+D)$-dimensional internal space $M^{4}$ $\otimes B^{D}$ will be denoted by $x^{M}=\left(x^{a}, x^{m}\right)$, where $x^{a}$ are the coordinates of $M^{4}$, and $x^{m}$ the coordinates of $B^{D}$. A local transformation of these coordinates, which leaves the metric $\gamma_{M N}$ invariant, can be written in the form

$$
\delta x^{M}=\delta \alpha^{A} K_{A} x^{M},
$$

where $\delta \alpha^{A}=\delta \alpha^{A}\left(x^{\mu}\right)$ are the infinitesimal parameters, whose components are written in the form

$$
\delta \alpha^{A}\left(x^{\mu}\right) \equiv\left(\delta \alpha^{a}, \delta \tilde{\alpha}^{i}\right)=\left(\delta \alpha^{a}, \frac{g}{\kappa c^{2}} \delta \alpha^{i}\right),
$$

and $K_{A}$ represent the generators of the transformations. These generators have the form

$$
K_{A}=K^{N}{ }_{A} \partial_{N},
$$

where the coefficients $K_{A}^{N}$ are the Killing vectors [14] associated with the infinitesimal isometries of the internal space $M^{4} \otimes B^{D}$. They form a set of $4+I$ linearly independent vectors for this space [1], and are given by

$$
K_{A}^{N}=\left(\begin{array}{cc}
\delta_{a}^{b} & 0 \\
0 & K^{n}
\end{array}\right),
$$

with $\delta^{b}{ }_{a}$ the Killing vectors of $M^{4}$, and $K^{n}{ }_{i}$ the Killing vectors of $B^{D}$. The generators are, consequently,

$$
K_{a}=\delta_{a}^{b} \partial_{b}=P_{a},
$$

which are the isometry generators of $M^{4}$, and

$$
K_{i}=K^{n}{ }_{i} \partial_{n}
$$

which are the isometry generators of $B^{D}$. The coordinate transformations, therefore, are given by

$$
\delta x^{a}=\delta \alpha^{a},
$$

and

$$
\delta x^{n}=\frac{g}{\kappa c^{2}} \delta \alpha^{i} K_{i}^{n} \equiv \frac{g}{\kappa c^{2}} \delta \xi^{n},
$$

with $\delta \xi^{n}=\delta \alpha^{i} K^{n}{ }_{i}$ the transformation parameter in the Killing basis.

The generators $K_{A}$ obey the algebra

$$
\left[K_{A}, K_{B}\right]=f_{A B}^{C} K_{C},
$$

where $f_{B C}^{A}$ are the (dimensional) structure constants, whose components are

$$
f^{A}{ }_{B C}=\left\{\begin{array}{lll}
f^{a}{ }_{b c}=0 & \text { for } & A, B, C=a, b, c, \\
\widetilde{f}^{i}{ }_{j k} \equiv \chi f^{i}{ }_{j k} & \text { for } & A, B, C=i, j, k .
\end{array}\right.
$$

The constant

$$
\chi=\kappa \frac{c}{\hbar},
$$

was introduced for dimensional reasons, and in such a way to yield correct forms for the field strengths and gauge transformations. We have thus the following commutation relations:

$$
\left[K_{a}, K_{b}\right]=0,
$$

and

$$
\left[K_{i}, K_{j}\right]=\chi f^{k}{ }_{i j} K_{k}
$$

The generalized derivative, covariant under the transformation (15), is

$$
\mathcal{D}_{\mu}=\partial_{\mu}+c^{-2} \mathcal{A}_{\mu}^{A} K_{A} .
$$

In fact, as a simple computation shows, its commutator gives rise to the generalized field strength,

$$
\left[\mathcal{D}_{\mu}, \mathcal{D}_{\nu}\right]=c^{-2} \mathcal{F}^{A}{ }_{\mu \nu} K_{A},
$$

where

$$
\mathcal{F}^{A}{ }_{\mu \nu}=\partial_{\mu} \mathcal{A}^{A}{ }_{\nu}-\partial_{\nu} \mathcal{A}^{A}{ }_{\mu}+c^{-2} f^{A}{ }_{B C} \mathcal{A}^{B}{ }_{\mu} \mathcal{A}^{C}{ }_{\nu} .
$$

Using the appropriate definitions, this expression is easily seen to yield the correct expressions for the gravitational and the Yang-Mills field strengths. We notice in passing that the tetrad field is given by the covariant derivative of $x^{a}$, the coordinates of the non-compact part $M^{4}$ of the fiber space:

$$
e^{a}{ }_{\mu}=\mathcal{D}_{\mu} x^{a}
$$

This means that this part of the fiber space presents the soldering property [15].

Now, from the covariance of $\mathcal{D}_{\mu}$ under the isometric transformation (15), we obtain the gauge transformation of the generalized potential:

$$
\delta \mathcal{A}^{A}{ }_{\mu}=-c^{2} \mathcal{D}_{\mu} \delta \alpha^{A} \equiv-c^{2} \partial_{\mu} \delta \alpha^{A}-f^{A}{ }_{B C} \mathcal{A}^{B}{ }_{\mu} \delta \alpha^{C} .
$$

For $A=a$, the usual transformation law for the (Abelian) gravitational gauge potential is obtained:

$$
\delta A^{a}{ }_{\mu}=-c^{2} \partial_{\mu} \delta \alpha^{a} .
$$


For $A=i$, it gives

$$
\delta A^{i}{ }_{\mu}=-\mathcal{D}_{\mu} \delta \alpha^{i} \equiv-\partial_{\mu} \delta \alpha^{i}-\frac{g}{\hbar c} f_{j k}^{i} A^{j}{ }_{\mu} \delta \alpha^{k},
$$

which is the usual non-Abelian gauge transformation. When $\delta \alpha^{a}=0$, therefore, the coordinate transformation (15) leads to a pure gauge transformation.

\section{GAUGE LAGRANGIAN AND FIELD EQUATIONS}

Considering the generalized field strength $\mathcal{F}^{A}{ }_{\mu \nu}$, we can write the unified Lagrangian density for the gauge field as

$$
\mathcal{L}=\frac{e}{16 \pi G}\left(\frac{1}{4} \mathcal{F}^{A}{ }_{\mu \nu} \mathcal{F}^{B}{ }_{\theta \rho} g^{\mu \theta} N_{A B}{ }^{\nu \rho}\right),
$$

where $e=\operatorname{det}\left(e^{a}{ }_{\mu}\right)$. The algebraic indices $A, B, C, \ldots$ are raised and lowered with the Cartan-Killing metric

$$
\eta_{A B}=\left(\begin{array}{cc}
\eta_{a b} & 0 \\
0 & \eta_{i j}
\end{array}\right),
$$

whose components related to the translation group coincide with the Lorentzian metric $\eta_{a b}$ of the Minkowski tangent space. The explicit form of the components of $N_{A B}{ }^{\nu \rho}$ are

$$
N_{a b}{ }^{\nu \rho}=\mathrm{A} \eta_{a b} e_{c}{ }^{\nu} e^{c \rho}+\mathrm{Be}_{a}{ }^{\rho} e_{b}{ }^{\nu}+\mathrm{Ce}_{a}{ }^{\nu} e_{b}{ }^{\rho},
$$

with $\mathrm{A}, \mathbb{B}, \mathrm{C}$ arbitrary parameters [9], which gives the Lagrangian of the gravitational sector, and

$$
N_{i j}{ }^{\nu \rho}=\eta_{i j} e_{c}{ }^{\nu} e^{c \rho},
$$

which gives the Lagrangian of the gauge sector. The difference in the form of $N_{A B}{ }^{\nu \rho}$ for the different sectors of the theory is directly related to the fact that, due to the presence of a tetrad field in the gravitational sector, the algebraic and spacetime indices of this sector are of the same type, and consequently there are additional ways of contracting the indices. Since there are no tetrads relating the algebraic and the spacetime indices in the gauge sector, only the usual contraction is present. For the specific choice of the parameters,

$$
\mathrm{A}=\frac{\mathrm{B}}{2}=-\frac{\mathrm{C}}{4}=1
$$

teleparallel gravity yields the so-called teleparallel equivalent of general relativity. In this case, the Lagrangian (34) becomes

$$
\mathcal{L}=\frac{c^{4} e}{16 \pi G} S^{\rho \mu \nu} T_{\rho \mu \nu}+\frac{\kappa^{-2} g^{2}}{16 \pi G} \eta_{i j}\left[\frac{e}{4} F^{i}{ }_{\mu \nu} F^{j \mu \nu}\right],
$$

where

$$
T_{\rho \mu \nu}=c^{-2} e_{a \rho} F_{\mu \nu}^{a} \equiv \Gamma_{\rho \nu \mu}-\Gamma_{\rho \mu \nu}
$$

is the torsion of the Weitzenböck connection $\Gamma^{\rho}{ }_{\mu \nu}$ $=e^{\rho}{ }_{a} \partial_{\nu} e^{a}{ }_{\mu}$, and $S^{\rho \mu \nu}$ is the tensor

$$
\begin{aligned}
S^{\rho \mu \nu}= & \frac{1}{4}\left(T^{\rho \mu \nu}+T^{\mu \rho \nu}-T^{\nu \rho \mu}\right) \\
& -\frac{1}{2}\left(g^{\rho \nu} T_{\theta}{ }^{\mu \theta}-g^{\rho \mu} T_{\theta}{ }^{\nu \theta}\right) .
\end{aligned}
$$

To obtain the correct form of the gauge Lagrangian, two conditions must be imposed on the second term of Eq. (38). The first one is that [6]

$$
\kappa^{2}=\frac{g^{2}}{16 \pi G} \equiv \frac{g^{2}}{\mathcal{G}^{2}} .
$$

The constant $\kappa$, therefore, is simply the relation between the gauge coupling constant $g$ and the gravitational coupling constant $\mathcal{G}$. The second condition concerns the relative signs between the gravitational and the Yang-Mills Lagrangians. In order to get the appropriate sign, it is necessary that

$$
\eta_{i j}=-\delta_{i j}
$$

Therefore, the Cartan-Killing metric (35) of the unified gauge group becomes

$$
\eta_{A B}=\left(\begin{array}{cc}
\eta_{a b} & 0 \\
0 & -\delta_{i j}
\end{array}\right) .
$$

With these conditions, we obtain

$$
\mathcal{L} \equiv \mathcal{L}_{\mathrm{G}}+\mathcal{L}_{\mathrm{YM}}=\frac{c^{4} e}{16 \pi G} S^{\rho \mu \nu} T_{\rho \mu \nu}-\frac{e}{4} F^{i}{ }_{\mu \nu} F_{i}{ }^{\mu \nu} .
$$

As is well known, up to a divergence, the first term of this Lagrangian is the teleparallel equivalent of the EinsteinHilbert Lagrangian of general relativity [10]. The second term, on the other hand, is the usual gauge Lagrangian in the presence of gravitation.

It is interesting to notice that, when the Cartan-Killing metric related to the (external) translation group $T_{4}$ is chosen to be $\eta_{a b}=\operatorname{diag}(+1,-1,-1,-1)$, the corresponding Cartan-Killing metric related to the (internal) Yang-Mills group $G_{\mathrm{YM}}$ has necessarily the form $\eta_{i j}=-\delta_{i j}$. If we had chosen the other possible convention for $\eta_{a b}$, that is, $\eta_{a b}$ $=\operatorname{diag}(-1,+1,+1,+1)$, the consistency condition would require that $\eta_{i j}=+\delta_{i j}$. Therefore, the teleparallel KaluzaKlein construction imposes a constraint between the CartanKilling metric convention adopted for the translation gauge group, and consequently for the Minkowski tangent spacesee the comment just after Eq. (35) — and that adopted for the Yang-Mills gauge group.

Performing a functional variation of $\mathcal{L}$ in relation to the components $A^{a}{ }_{\tau}$, and using the definition of the Weitzenböck connection $\Gamma_{\mu \nu}^{\rho}$, we obtain the gravitational field equation

$$
\partial_{\sigma}\left(e S_{\lambda}^{\tau \sigma}\right)-\frac{4 \pi G}{c^{4}} e t_{\lambda}^{\tau}=\frac{4 \pi G}{c^{4}} e \Theta_{\lambda}^{\tau},
$$

where 


$$
e t^{\tau}{ }_{\lambda}=\frac{c^{4} e}{4 \pi G} \Gamma_{\nu \lambda}^{\mu} S_{\mu}{ }^{\tau \nu}-\delta^{\tau}{ }_{\lambda} \mathcal{L}_{\mathrm{G}}
$$

stands for the teleparallel canonical energy-momentum pseudo-tensor of the gravitational field [16]. The source of the field equation (44) is the energy-momentum tensor of the Yang-Mills field in the presence of gravitation, which is defined by

$$
e \Theta^{\tau}{ }_{\lambda} \equiv-c^{-2} e^{a}{ }_{\lambda} \frac{\delta \mathcal{L}_{\mathrm{YM}}}{\delta A^{a}{ }_{\tau}}=-e^{a}{ }_{\lambda} \frac{\delta \mathcal{L}_{\mathrm{YM}}}{\delta e^{a}{ }_{\tau}} .
$$

Finally, variation of $\mathcal{L}$ with respect to the components $A^{i}{ }_{\mu}$ yields the Yang-Mills equation in the presence of gravitation,

$$
\partial_{\mu}\left(e F^{i \mu \nu}\right)-\frac{g e}{\hbar c} j^{i \nu}=0,
$$

where

$$
j^{i \nu}=-f_{j k}^{i} A_{\mu}^{j} F^{k \mu \nu}
$$

stands for the (pseudo) current of the Yang-Mills field [17]. We mention in passing that the teleparallel field equation (44) is an equation written in terms of the Weitzenböck connection only. It can alternatively be written in terms of the Levi-Civita connection, in which case it reduces to the general relativity Einstein's equation. The teleparallel field equation (44), however, has the advantage of presenting the same formal structure of the Yang-Mills equation.

\section{MATTER FIELDS}

The geometrical structure underlying every gauge theory exists independently of the presence or not of gauge fields. For example, in the teleparallel Kaluza-Klein theory, the non-compact four-dimensional part of the fiber, which is given by the tangent Minkowski space, is a geometrical structure that is always present independently of the presence or not of a gravitational gauge field. The same is true of the $D$-dimensional compact part of the fiber in relation to the corresponding Yang-Mills gauge field. It should be noticed that the fiber space of teleparallel Kaluza-Klein theories corresponds to the ground state spacetime of ordinary KaluzaKlein theories. In these theories, the transition from a higherdimensional theory to the effective four-dimensional theory is made with the help of an harmonic expansion around the ground state, whose excitations represent the field variables of the model. As a consequence, an infinite spectrum of particles is obtained. In particular, the lowest order excitations have vanishing mass, giving rise to the massless sector of the emerging gauge theory.

On the other hand, in the teleparallel Kaluza-Klein theories, all dynamical variables are functions of the fourdimensional spacetime points. Furthermore, the action functional and the field equations are written in the fourdimensional spacetime, and not in the higher-dimensional fiber space. This means that no dimensional reduction is necessary, no harmonic expansion around the ground state has to be performed, and consequently the field variables of the model cannot be represented by excitations. In fact, like in any other gauge theory, the basic fields are represented by gauge potentials given a priori, which are the basic ingredients for the construction of gauge theories.

Now, comes the question on how the dynamical variables of the theory depend on the coordinates $x^{M}$ of the fiber space. Concerning the coordinates $x^{a}$ of the non-compact four-dimensional part $\left(M^{4}\right)$ of the fiber, as this space is soldered to spacetime, and as all dynamical variables are functions of the spacetime coordinates $x^{\mu}$, the dependence of these variables on $x^{a}$ has necessarily to be through the argument of the dynamical variables. Concerning the coordinates $x^{m}$ of the compact $D$-dimensional part $\left(B^{D}\right)$ of the fiber, as a change in $x^{m}$ must correspond to a change, not in the argument, but in the components of the field variable, the dependence of any dynamical variable on $x^{m}$ will be analogous to the dependence on the gauge parameter in a gauge theory. This is a direct consequence of the fact that the isometry transformations of $B^{D}$ are ultimately equivalent to internal gauge transformations. Accordingly, the dependence of the matter field $\Psi$ on the coordinates $x^{m}$ can be written in the form

$$
\Psi\left(x^{m}\right)=\exp \left[i \chi \beta_{n} x^{n}\right] \psi,
$$

where $\chi$ is defined in Eq. (25), and $\beta_{n}$ are parameters related to the geometry of the compact manifold $B^{D}$. In addition, as a change in $x^{m}$ is related to a gauge transformation, $\beta_{n}$ must necessarily assume values in the Lie algebra of the gauge group. In other words, $\beta_{n}=\beta_{n}{ }^{j} \mathcal{T}_{j}$, with $\mathcal{T}_{j}$ a matrix representation of the Lie algebra generators. In fact, according to Eq. (47), the action of the (derivative) isometry generators $K^{n}{ }_{i} \partial_{n}$ turns out to be equivalent to the action of the (multiplicative) matrix generators $i \chi K^{n}{ }_{i} \beta_{n}$. This means that it is possible to relate $i \chi K^{n}{ }_{i} \beta_{n}$ to another realization of the generators of the gauge group. As already said, this possibility is a direct consequence of the fact that the (internal) gauge transformations are obtained as the isometries of $B^{D}$.

Let us explore better this point. Under the coordinate transformation (15), a matter field $\Psi$ changes according to

$$
\delta \Psi=\delta \alpha^{A} K_{A} \Psi \equiv \delta \alpha^{a} \partial_{a} \Psi+\frac{g}{\kappa c^{2}} \delta \alpha^{i} K^{n}{ }_{i} \partial_{n} \Psi
$$

By using Eq. (47), we see that

$$
\partial_{n} \Psi=i \chi \beta_{n} \Psi=i \frac{\kappa c}{\hbar} \beta_{n} \Psi
$$

where use has been made of Eq. (25). Substituting into the transformation (48), it becomes

$$
\delta \Psi=\delta \alpha^{a} \partial_{a} \Psi+\frac{i g}{\hbar c} \delta \alpha^{i} K^{n}{ }_{i} \beta_{n} \Psi
$$

On the other hand, we have already seen that $K_{i}=K^{n}{ }_{i} \partial_{n}$ satisfy the commutation relations (27), that is

$$
\left[K_{i}^{n} \partial_{n}, K^{m}{ }_{j} \partial_{m}\right] \Psi=\chi f^{k}{ }_{i j} K^{n}{ }_{k} \partial_{n} \Psi \text {. }
$$


As the Killing vectors $K^{n}{ }_{i}$ depend on $x^{m}$ in the same manner as $\Psi$ does, it is an easy task to verify that

$$
\left[i K_{i}^{n} \beta_{n}, i K^{m}{ }_{j} \beta_{m}\right] \Psi=f^{k}{ }_{i j} i K^{n}{ }_{k} \beta_{n} \Psi .
$$

This means that

$$
T_{i}=i K^{n}{ }_{i} \beta_{n} \equiv i K^{n}{ }_{i} \beta_{n}{ }^{j} \mathcal{T}_{j}
$$

can be identified as another realization of the (antiHermitian) Lie algebra generators. With this identification, the transformation (50) acquires the form

$$
\delta \Psi=\delta \alpha^{a} P_{a} \Psi+\frac{g}{\hbar c} \delta \alpha^{i} T_{i} \Psi
$$

which is in fact a gauge transformation of matter fields.

The covariant derivative of $\Psi$ is defined by

$$
\mathcal{D}_{\mu} \Psi=\partial_{\mu} \Psi+\mathcal{A}^{A}{ }_{\mu} \frac{\delta \Psi}{\delta \alpha^{A}} .
$$

Substituting the transformation (31), and using the appropriate identifications, it becomes

$$
\mathcal{D}_{\mu} \Psi=e^{a}{ }_{\mu} \partial_{a} \Psi+\frac{g}{\hbar c} A^{i}{ }_{\mu} T_{i} \Psi
$$

which is the usual expression of the gauge covariant derivative in the presence of gravitation. Defining $A_{\mu}^{i}=A_{a}^{i} e^{a}{ }_{\mu}$, it can be rewritten in the form

$$
\mathcal{D}_{\mu} \Psi=e^{a}{ }_{\mu} \mathcal{D}_{a} \Psi,
$$

where $\mathcal{D}_{a} \Psi$ is the gauge covariant derivative in Minkowski spacetime.

\section{FINAL REMARKS}

Replacing the general relativity paradigm by a gauge paradigm, and making use of the teleparallel description of gravitation, which corresponds to a gauge theory for the translation group, we have succeeded in constructing a teleparallel version of the non-Abelian Kaluza-Klein theory. In other words, we have succeeded in unifying, in the Kaluza-Klein sense, teleparallel gravitation with Yang-Mills type theories. The resulting model turns out to be a gauge theory for the group $T_{4} \otimes G_{\mathrm{YM}}$, with the fiber space given by $M^{4} \otimes B^{D}$, where $M^{4}$ is the Minkowski tangent spacetime, and $B^{D}$ is the manifold associated with the Yang-Mills gauge group $G_{\mathrm{YM}}$. In this model, the translational gauge transformation arises as the isometries of the non-compact fourdimensional part of the fiber, which is always a Minkowski spacetime $M^{4}$, whereas the non-Abelian gauge transformations arise as the isometries of the compact $D$-dimensional part of the fiber, which is the part related to the internal gauge symmetry.

As in the Abelian case [11], the teleparallel equivalent of the non-Abelian Kaluza-Klein model turns out to be much more natural than the ordinary Kaluza-Klein model. In fact, in the teleparallel model both gravitational and Yang-Mills type fields are described by a gauge theory, with the YangMills field strength appearing as extra gauge components of torsion, the field strength of teleparallel gravity. This means that the gravitational and the Yang-Mills field strengths are different components of a unique tensor. Another interesting point concerns the relation between geometry and gauge theories. According to ordinary Kaluza-Klein models, gauge theories emerge from higher-dimensional geometric theories as a consequence of the dimensional reduction process. According to the teleparallel Kaluza-Klein approach, however, gauge theories are the natural structures to be introduced, the four-dimensional geometry (gravitation) emerging from the non-compact sector of the fiber space. In fact, only this sector presents the soldering property [15], and can consequently give rise to a tetrad field, which is the responsible for the geometrical structure (either metric or teleparallel) induced in spacetime. Furthermore, as the gauge theories are introduced in their original form-they do not come from geometry - the unification, though not trivial, turns out to be much more natural and easier to be performed.

An important characteristic of the ordinary non-Abelian Kaluza-Klein model is that the metric (1) is not a solution of the higher-dimensional Einstein equations as these equations cannot have solutions of the form $M^{4} \otimes B^{D}$. This is related to the fact that the $D$-dimensional internal space is in general curved, leading then to difficulties for defining the ground state (vacuum) of the higher-dimensional gravitational field. These models, therefore, require an initial non-compact (4 $+D$ )-dimensional spacetime, and a subsequent compactification scheme for the $D$ extra dimensions. One way of solving this problem is to introduce extra matter fields in the form of a higher-dimensional energy-momentum tensor, so that a spontaneous compactification of the extra dimensions is achieved [18]. Another solution was that provided by Freund and Rubin [19] in eleven-dimensional supergravity, where not only is there compactification, but the space naturally separates in $(4+7)$ dimensions. On the other hand, since in the teleparallel Kaluza-Klein model the gauge theories are not obtained from the geometry, but introduced in their original forms, the fiber space of these theories can be assumed to present a compact sub-manifold $B^{D}$ from the very beginning. In other words, the compactification problem does not exist for these theories. In addition, as both the action and the field equations are always written in the fourdimensional spacetime, and not in the higher-dimensional fiber space, no dimensional reduction is necessary, and consequently no expansion of the dynamical variables in terms of the complete set of harmonics of $B^{D}$ has to be performed. As a consequence, the infinite spectrum of new particles is absent, strongly reducing the redundancy present in ordinary Kaluza-Klein theories. A similar achievement has already been obtained by a modified Kaluza-Klein theory in which the internal coordinates are replaced by generators of a noncommutative algebra [20]. In this model, no truncation to eliminate extraneous modes is necessary as only a finite number of them is present.

Finally, as a last remark, let us take the internal coordinate transformation (22), and substitute on it the value of $\kappa$, given 
by Eq. (40). As $B^{D}$ is compact, this transformation can be written in the form

$$
\delta x^{n}=\rho \delta \theta^{n},
$$

where

$$
\rho=l_{P} \equiv\left(\frac{G \hbar}{c^{3}}\right)^{1 / 2}
$$

is the length scale associated with the compact internal submanifold, and

$$
\delta \theta^{n}=\left(\frac{16 \pi}{\hbar c}\right)^{1 / 2} \delta \xi^{n}
$$

is the angular coordinate associated with $x^{m}$. We see in this way that the teleparallel Kaluza-Klein model defines a natu- ral length scale for the compact part of the fiber space, given by the Planck length. In the specific case of the ordinary Abelian Kaluza-Klein theory, the radius of the fifth dimension can only be inferred from the value of the elementary electric charge. Since the teleparallel model yields a natural length scale, we can reverse the argument and use this length to calculate the elementary electric charge. Furthermore, as is well known, a length of the order of the Planck length, like the $\rho$ above, through the application of the Bohr-Sommerfeld quantization rule to the periodic motion in the fifth dimension, gives the correct value for the elementary electric charge.

\section{ACKNOWLEDGMENTS}

The authors would like to thank FAPESP-Brazil, CAPESBrazil and CNPq-Brazil for financial support.

(1997).

[11] V.C. de Andrade, L.C.T. Guillen, and J.G. Pereira, Phys. Rev. D 61, 084031 (2000).

[12] W. Drechsler, Phys. Lett. 90B, 258 (1980).

[13] S.K. Wong, Nuovo Cimento A 65, 689 (1970).

[14] See, for example, S. Weinberg, Gravitation and Cosmology (Wiley, New York, 1972).

[15] R. Aldrovandi and J. G. Pereira, An Introduction to Geometrical Physics (World Scientific, Singapore, 1995).

[16] V.C. de Andrade, L.C.T. Guillen, and J.G. Pereira, Phys. Rev. Lett. 84, 4533 (2000).

[17] P. Ramond, Field Theory: A Modern Primer, 2nd ed. (Addison-Wesley, Redwood, 1989).

[18] E. Cremmer, Z. Horvath, L. Palla, and J. Scherk, Nucl. Phys. B127, 57 (1977).

[19] P.G.O. Freund and M.A. Rubin, Phys. Lett. 97B, 233 (1980).

[20] J. Madore and J. Mourad, lecture given at the 5th Hellenic School and Workshop on Elementary Particle Physics, hep-th/ 9601169.
[9] K. Hayashi and T. Shirafuji, Phys. Rev. D 19, 3524 (1979).

[10] V.C. de Andrade and J.G. Pereira, Phys. Rev. D 56, 4689 